\title{
Retrospective Drug Use pattern of Antibiotics in Pediatric Ward of Shenan Gibe Hospital, Oromia Region, Ethiopia
}

\section{Achalu $\mathrm{T}^{1}$ and Mensa $\mathrm{M}^{{ }^{2}}$}

${ }^{1}$ B.Pharm, Jimma University, Ethiopia

${ }^{2}$ B.Pharm MSC, Clinical pharmacist, Arba Minch College of health Sciences, Ethiopia

${ }^{*}$ Corresponding author: Mensa M, B.Pharm, MSC. Clinical pharmacist, Arba Minch College of health Sciences, Ethiopia, Fax: +251046881147, Tel: +251937170976, E-mail: mendemensa@gmail.com

Citation: Barghouthi Achalu T, Mensa M (2017) Retrospective Drug Use pattern of Antibiotics in Pediatric Ward of Shenan Gibe Hospital, Oromia Region, Ethiopia. J Antibio Res 1(1): 106. doi: 10.15744/25745980.1.106

Received Date: February 28, 2017 Accepted Date: March 31, 2017 Published Date: April 05, 2017

\begin{abstract}
Background: Antibiotics are one of the most widely used groups of drugs. The development of antimicrobial drugs represents one of the most important advances in therapeutics. Irrational and uncontrolled use of these agents both in developing and developed countries has resulted in an evolution of resistant strains in addition to poor health outcome. Despite this fact antimicrobial agents are vastly over prescribed in inpatient setting and the availability of antimicrobial agents without prescription in many developing countries. The use antimicrobial agents in Infants and children have become a routine practice for the treatment of pediatric illness and it needs study to show the status and pattern of antimicrobial in this age group is critical hence they are most vulnerable population groups to counteract illness.

Objective: To assess the pattern of antibiotics use among hospitalized Pediatric patients in pediatric ward of Shenen Gibe Hospital, May 2015.

Methods: A retrospective cross-sectional study design was used to collect data from patient medical history records from previous one year data and Data was collected by using pre-tested structured tool which is adapted from review of different literatures.

Result: Three hundred twenty three pediatrics medical history cards with antibiotics were reviewed in this study. Majority of participants $188(58.2 \%)$ were males and $197(61 \%)$ and belongs to age group 0-4 years with mean age of 3.5 years. Regarding duration of hospitalization for 200 (61.9\%) was 5 to 9 days with median duration of 5.7 days. Majority of patients 251(77.7\%) were treated empirically. Pneumonia was single most common reason for hospitalization which accounts 125 (38.7\%) followed by Sepsis 49 (15.2\%) and acute gastro enteritis 41(12.7\%). The most commonly used antibiotic combinations were Ceftriaxone + Gentamicin 97(32.8\%). Antibiotics were prescribed with correct dose for 294(91\%) and correct frequency for 280(86.7\%) and 316(97.6\%) were treated with injections.

Conclusion and Recommendation: In our study use of parenteral antibiotics was relatively higher and almost all treatments were empiric and increased error of dosing; frequency and duration of treatment with combination of therapy this call for the governing body should disseminate relevant information to the hospital to raise the level of awareness about rational use of drugs, prescribing and dispensing of drugs through a continuous in services training, seminars and workshops to reduce use of combined systemic broad spectrum antibiotics which could lead to selection of naturally resistant strains to emerge unless intervened now "No action today: No cure tomorrow."

Keywords: Antibiotics; Hospitalization; pediatrics patient; Shenan Gibe; Ethiopia

List of Abbreviations: DUE: Drug Use Evaluation; DUR: Drug utilization review; PMHR: Patient Medical History record; STG: Standard Treatment Guideline; ADR: Adverse Drug Reaction; AFI: Acute Febrile Illness; URTI: Upper Respiratory Tract Infection; AZ: Azithromycin; CPX: Ciprofloxacin; LFX: Levofloxacin; RDU: Rational Drug use
\end{abstract}

\section{Introduction}

Antibiotics are substances or compounds which have the capacity to inhibit the growth of or to kill microorganism. Antibiotics have saved millions of lives since their first appearance of penicillin in 1940s, even though more people are dying from infectious disease that were curable but for which we no longer have the right treatment due to Antimicrobial resistance, which is a global problem, is particularly pressing in developing countries, where infectious disease burden is very high [1].

The evolving public health threat of antimicrobial resistance (AMR) is driven by both appropriate and inappropriate use of antiinfective medicine for human and animal health and food production together with inadequate measures to control the spread 
of infections. Recognizing these crisis due to AMR, several nations in the worldwide have developed strategies to counteract it through, a six-point policy package; (1) commit to a comprehensive, financed national plan with accountability and civil society engagement, (2) strengthen surveillance and laboratory capacity, (3) ensure uninterrupted access to essential medicines of assured quality, (4) regulate and promote rational use of medicines in animal husbandry and to ensure proper patient care, (5) enhance infection prevention and control, and (6) foster innovations and research and development of new tools [2].

Continuous availability of essential drugs including antibiotics of proven safety, efficacy, quality and acceptability of affordable price compounded by rational drug use is indispensable for health care delivery [3-5].

Infants and children are the most vulnerable population groups to counteract illness. The use of antimicrobial agents especially antibiotics has become a routine practice for the treatment of pediatric illness. At the same time antimicrobial agents are over prescribed in inpatient setting and the availability of antimicrobial agents without prescription in many developing countries has been facilitating the development of resistance [6-8].

Antibiotics represent approximately $30 \%$ of acute care hospitals' drug expenditure and they are prescribed for 20-50\% of inpatients. WHO estimates more than half of all medicines are prescribed, dispensed inappropriately, half of all patients fail to take them correctly and 22-65\% of antibiotic prescriptions are inappropriate [9-12].

Pediatric population differs in physiology; pharmacokinetics and pharmacodynamics from adult populations. Markets for children's medicines tend to be small and the range of doses used may be wide for any drug formulation because many drugs prescribed widely for infants and children are not available in suitable dosage forms, leading to a lack of attention to pediatric medicines $[13,14]$.

Infectious diseases cause one in every six deaths worldwide and emerging antimicrobial resistance in bacteria threatens management of bacterial infections. In Sub-Saharan, studies on antimicrobial resistance have been done, but, by large, the issue has received far too little attention [15].

Ethiopia has designed national strategic for Containment of Antimicrobial Resistance to facilitate efforts in minimizing the morbidity and mortality due to resistant infections and preserving the effectiveness of antimicrobial medicines. Even though there are indications on the misuse of AMs by health care providers', unskilled practitioners and animal husbandry and drug users. These coupled with rapid spread of resistant bacteria and inadequate surveillance has exacerbated the problem [16].

Knowledge of drug administration in children and infants lags behind that of adults for many reasons including developmental differences that affect the pharmacodynamic and pharmacokinetic profiles of drugs, ethical and financial reasons, research capabilities, and regulatory guidelines and constraints [17].

Irrational drug use, especially antimicrobials in a pediatric population, has become a commonly noted practice. A study in the USA and Canada has shown that $50 \%$ and $85 \%$ of antibiotics, respectively, were prescribed inappropriately to children [18].

Studies have indicated that children are prescribed drugs frequently, with a mean number of drugs as high as 5.5. Another study conducted on pediatric drug prescribing in trinidad revealed that $44 \%$ of patients had received one or more antibiotics and pediatric patients received $70.8 \%$ antibiotics. Most frequently prescribed antibiotics to children were Ampicillin, Chloramphenicol and Gentamicin [19]. Retrospective drug utilization evaluation in pediatric patients in USA showed that large percentages (30\%$50 \%$ ) of antibiotics administered in the USA are given to prevent infection rather than to treat established disease [20].

Study conducted in Mc-Gann Teaching Hospital showed that the average number of antibiotics prescribed per patient was 2.14 \pm 0.62 and $92 \%$ of patients received multiple antibiotics. The most commonly prescribed single antibiotic was third generation cephalosporin. Most common prevalence of disease among the study was acute GE and it is treated commonly by Cefixime and Gentamicin. Most 75\% of the antibiotic prescriptions were based on clinical diagnosis, not on culture report [21].

Study from Indonesia in paediatric wards indicated that the antibiotics were given to $48.7 \%$ of the patients. The greatest user of antibiotics was the age group of 1-5 year-old, $50 \%$ of patients had been given single antibiotic and the rest given combined antibiotics. Single antibiotics used were Cefotaxime (49.4\%), Amoxicillin (20.7\%), and Ampicillin (11.55\%). Combined antibiotics used were Ampicillin + Chloramphenicol (34.5\%), Cotrimoxazole (21.8\%), Cefotaxime + Amikacin (5.7\%), and Cefotaxime + Cotrimoxazole (5.7\%) [22].

Study conducted in Jimma university specialized hospital that the major medical conditions for which antibiotics were prescribed include severe pneumonia (31.23\%) and late onset neonatal sepsis with meningitis (20.60\%). The average number of antibiotics per patient was 2.17 . Only one antibiotic was prescribed in $22.80 \%$, two antibiotics in $51.47 \%$ (highest), three antibiotics in $16.91 \%$ $\&$ four or more than four antibiotics in $8.82 \%$ were prescribed. The most commonly prescribed multiple antibiotic was Ampicillin plus Gentamicin (21.9\%) followed by Ceftriaxone \& Gentamicin (18.1\%) [23].

The study from Bishoftu Hospital explored that among the most commonly prescribed antibiotics, Ceftriaxone accounted for $73(43.50 \%)$ followed by Gentamicin 43 (25.60\% and also the most common reasons for which drugs was prescribed were pneumonia followed by gastroenteritis which made up $72(56.25 \%)$ and $12(9.40 \%)$ of the indications respectively. Out of the 242 total number of medication prescribed, parenteral route was accounted for $201(83.1 \%)$ and the proportion of drugs prescribed in generic name was high which was 234 (97.5\%) [24]. 
A study done in Hawassa University Referral Hospital showed that the mean number of antibiotics prescribed per-prescription was $1.18 \pm 0.813$. The mean number of injection prescribed per-prescription was $1.48 \pm 0.766$. Seven hundred fifty (64.2\%) of the prescriptions were mono drug prescriptions. The most frequently prescribed single antibiotic was penicillin $\mathrm{G}$ crystalline 146 (28.4\%), followed by Ceftriaxone 128 (24.9\%). Four hundred sixteen (35.6\%) of prescriptions were multiple antibiotics and from these the combination of Ampicillin and Gentamicin took the largest portion with 113(27\%), followed by Chloramphenicol and Cloxacillin 60(14.4\%) [25].

There was no study conducted in the study area on the same and related topic, so the present study was intend to address issues about rational use of antibiotics in the study area. More ever this study will provide preliminary data to further investigators who are interested to conduct stronger studies. The finding may also call for attention of concerned bodies to make decisions and take measure in the spirit of improving drug use evaluation in particular antibiotics in Shenan Gibe Hospital.

\section{Objective of the Study}

\section{General Objective}

$\checkmark$ To assess the pattern of antibiotics use among hospitalized Pediatric patients in pediatric ward of Shenen Gibe Hospital, May 2015.

\section{Specific objectives}

$\checkmark$ To identify reasons of hospitalization among pediatric patients at Shenan Gibe district Hospital

$\checkmark$ To assess antibiotic use pattern among hospitalized pediatric patients at Shenan Gibe district Hospital

\section{Method and Materials}

\section{Study Area and Period}

The study was conducted in Shenan Gibe Hospital from May $1^{\text {st }}-27^{\text {th }}, 2015$. Shenen Gibe Hospital is one of district hospitals found in Oromia regional state and located in jimma town, which about $360 \mathrm{~km}$ to south west of the capital city, Addis Ababa. The hospital provides primary and secondary care to peoples Jimma town refers advanced cases to Jimma university specialized hospital.

\section{Study design}

Hospital based retrospective descriptive cross-sectional study design was used.

\section{Population}

Source population: All pediatrics patient medical history cards in Shenan Gibe hospital.

Study population: All pediatrics patient medical history Cards with antibiotics in Shenan Gibe Hospital at inpatient ward.

\section{Eligibility criteria}

\section{Inclusion criteria}

$\rightarrow$ All complete pediatric patients' medical history cards with at least one antibiotic.

$\rightarrow$ Pediatric patient admitted for 24 hour or more

\section{Exclusion criteria}

$\Delta$ Medical history record cards of patients who are admitted for less than $24 \mathrm{hrs}$.

$\checkmark$ Patient received topical antibiotics like ointment and drops.

$\triangle$ Patient received ant TB drugs only.

\section{Sample size determination}

The required sample size was calculated using single population proportion formula by considering 50\% proportion correct antibiotic use in hospitals because there is no similar study conducted in the study area which was published. 


$$
n=\frac{Z^{2} p q}{d^{2}} \quad n=\frac{(1.96) *(1.96) * 0.5(0.5)}{(0.05) *(0.05)}=384
$$

\section{Where}

$\mathrm{n}=$ the required samples size calculated by using single population proportion formula.

$\mathrm{Z}=$ Standard score corresponding to $95 \% \mathrm{CI}=1.96$

$\mathrm{P}=$ Assumed proportion of correct antibiotic use to be $\mathrm{p}=0.5$ for maximum sample.

$\mathrm{q}=1-\mathrm{P}=0.5$

$\mathrm{d}=$ the margin of error tolerable, i.e. $5 \%$

The average number total Pediatric admissions per year were 1692 patients and hence less than 10,000 therefore it is corrected using finite population correction formula.

Using this sampling size estimation method, a total sample size of 313 was obtained. To compensate for incomplete data $5 \%$ of the size was added and a total of 329 patient's cards were taken from study population.

\section{Corrected sample size}

$$
n=\frac{\text { no }}{1+\text { no } / \mathrm{N}}=312.98 \approx \mathbf{3 1 3}
$$

Finally $10 \%$ was added for non-response rate.

$=313+15.6=239$

\section{Sampling technique}

Systematic random sampling technique was used to select pediatrics patient medical records with antibiotics. Every fifth pediatrics patient medical records was selected from average total 1692 pediatrics patient medical records of one year. The first number i.e. number 3 was selected randomly using lottery method.

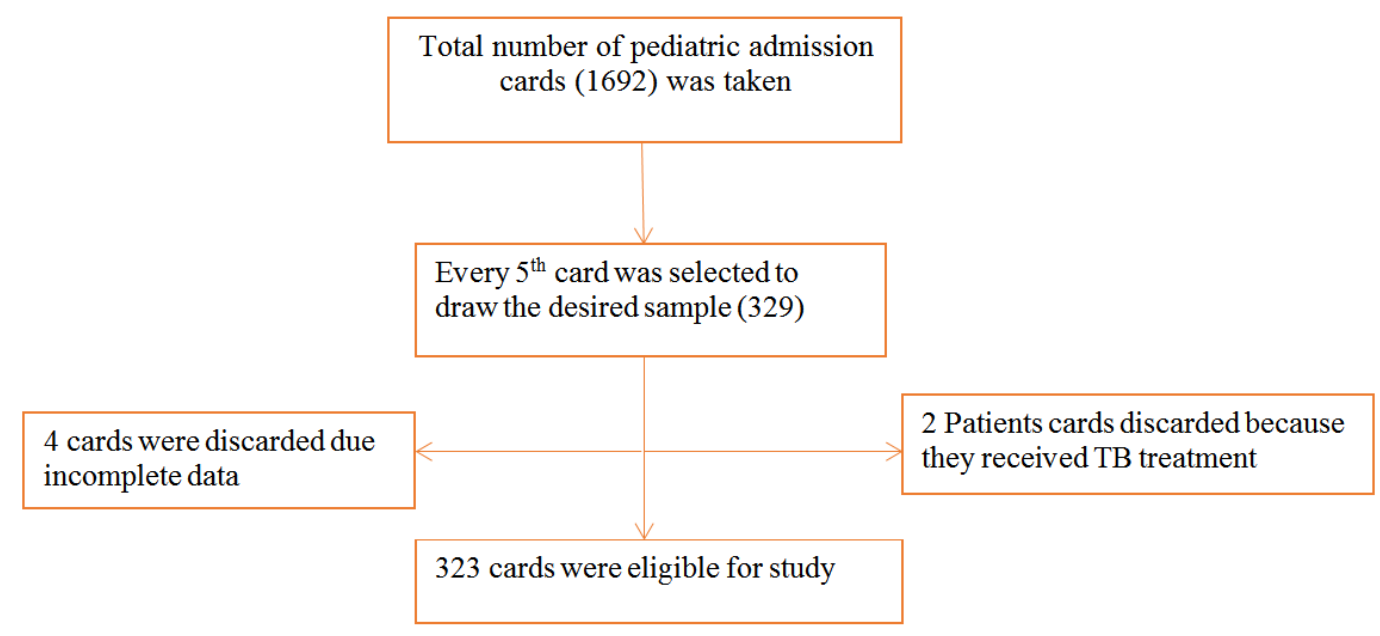

\section{Study Variables}

Figure 1: Diagrammatic representation of sampling technique

\section{Dependent variable}

$\checkmark$ Pattern of antibiotic use

\section{Independent variables}

$\triangleright$ Sex; Age

$\triangleright$ Reason of treatment

$>$ Diagnosis

$\triangleright$ Drug use 


\section{$\rightarrow$ Duration of treatment \\ $\Delta$ Frequency of administration}

\section{Data Collection procedures}

\section{Data Collection Instruments and Methods}

Structured data collected tools were developed after reviewing different literatures and pretested in 5\% of actual sample size that is 12 patient cards were used to check the consistency and completeness of the tool. Data was collected by four $5^{\text {th }}$ year clinical pharmacy students after orientation was given by principal investigators.

\section{Data Quality Assurance}

Questionnaires were prepared in English. The English versions was used for data collection after pretesting on 5\% (12) of the actual sample size in Jimma university specialized Hospital pediatrics ward to ensure consistency of tool and possible amendments were made to the questionnaire based on findings. Four $5^{\text {th }}$ year clinical pharmacy students for data collection and one clinical pharmacist for working in the hospital for supervision were given orientation before data collection about principles to follow during data collection and the contents of data collection format for one day by the principal investigators. Continuous follow up and supervision was made by the principal investigator throughout the data collection period.

\section{Data Processing and Analysis}

The collected data was checked for completeness and consistency by principal investigators on daily basis at the spot during the data collection time. Then data entry was made using Epi-data 3.1 software. After data processing, analysis was made using SPSS version 20.0. A summary descriptive statistics was computed for variables and pattern of drug use. The result was presented by using tables, figures and texts as based on type of data.

\section{Definition of terms}

Antibiotics: Chemotherapeutic agents with activity against microorganisms.

Definitive therapy: Treatment based on laboratory finding suggesting specific pathogen.

Drug use evaluation: An ongoing systematic process designed to maintain the appropriate and effective use of drugs.

Empiric therapy: Treatment based on sign and symptom without confirming pathogen by laboratory.

Empiric treatment: Antibiotics administration without laboratory identification of sensitive bacterial pathogen.

Incomplete data: Patient card lacking either one or more of the following (Age, sex, drug dose, duration of treatment and diagnosis).

Infants: Individuals with Age less than 1 year

Injections per prescription: Number of antibiotic injections per a given prescription.

Judicious use of drugs: Using drugs with a sound judgment according to present and validated treatment protocol.

Kinetic treatment: Treatment undertaken after definitive identification of the causative agent.

Neonate: Individuals with birth to 28 days

Nosocomial infection: An infection that is acquired in the hospital settings.

Pediatrics: Individuals with age up to 14 years

Rational use of drugs: A process which involves appropriate prescribing, dispensing and patient use of drugs.

Resistance: Refers to irresponsiveness of microorganism to the antibiotics through developing their own mechanism.

\section{Ethical Consideration}

Letter was obtained from the Ethical Review Board of Jimma University, College of Public Health and Medical Science. Permission letters to conduct the study was obtained from, Jimma Zone, health department and Shenan Gibe Hospital administration.

\section{Results}

\section{Socio-Demographics Characteristics}

Three hundred twenty three pediatrics medical history cards with antibiotics were reviewed in this study. Majority of participants 188 (58.2\%) were males and 197 (61\%). belongs to age group 0-4 years with mean age of 3.5 years. Regarding duration of hospitalization for 200 (61.9\%) was 5 to 9 days with median duration of 5.7 days (Table 1). 


\begin{tabular}{|c|c|c|}
\hline Socio-demographics characteristics & Frequency & $\%$ \\
\hline Sex & & \\
\hline Male & 188 & 58.2 \\
\hline Female & 135 & 41.8 \\
\hline Age category of pediatric & & \\
\hline 0-4 years & 197 & 61.0 \\
\hline 5-9 years & 89 & 27.6 \\
\hline 10-14 years & 37 & 11.5 \\
\hline Duration of hospitalization & & \\
\hline 1-4 days & 71 & 22.0 \\
\hline 5-9 days & 200 & 61.9 \\
\hline 10-14 days & 35 & 10.8 \\
\hline 15-19 days & 6 & 1.9 \\
\hline 20+ days & 11 & 3.4 \\
\hline
\end{tabular}

Table 1: Socio-demographics characteristics of hospitalized pediatrics patients at Shenan Gibe Hospital, May, 2015 ( $n=323)$

\section{Types of diagnosis and Drug use pattern}

From the total 323 pediatric patients included in this study, 251 (77.7\%) had got empirical diagnosis for their illness during their hospitalization and $73(22.6 \%)$ hospitalized pediatrics had got laboratorial diagnosis for their illness. Concerning purpose of therapy, all the prescribed drugs were prescribed as empiric treatment for $245(75.9 \%)$ and definitive treatment for 78 (24.1\%) respectively (Figure 2).

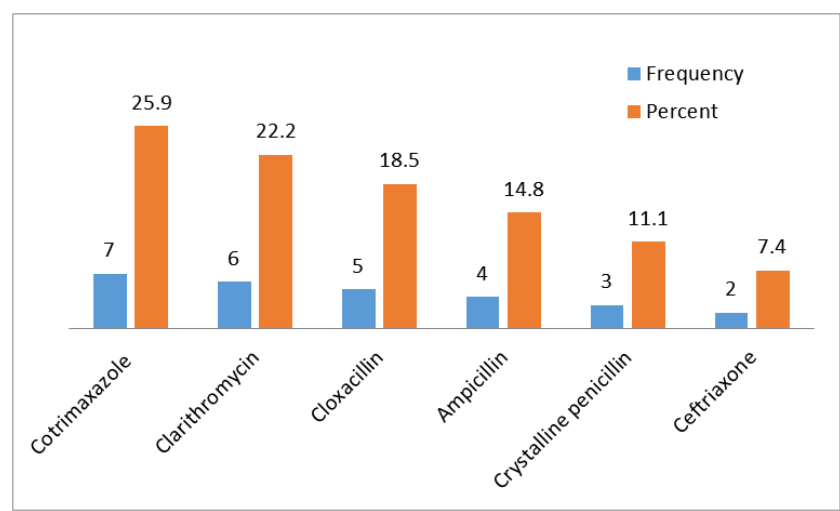

Figure 2: Frequency Distribution single antibiotics used in pediatric Ward at Shenan Gibe Hospital, May 2015 (n=27).

The number of diseases diagnosed per individual patient 292 (90.4\%) had single diagnosis and 31 (9.6\%) had two illnesses. Pneumonia was single most common reason for hospitalization which accounts 125 (38.7\%) followed by Sepsis 49 (15.2\%) and acute gastro enteritis 41 (12.7\%). With regard to combined illnesses Pneumonia and Malnutrition accounted 42 (56.8\%) followed by malaria and meningitis 23 (31.1\%) and sepsis and malnutrition 9(12.2\%) (Table 2).

\begin{tabular}{|c|c|c|}
\hline Diagnosis & Frequency & Percent \\
\hline Pneumonia & 125 & 38.7 \\
\hline Sepsis & 49 & 15.2 \\
\hline Acute gastro enteritis & 41 & 12.7 \\
\hline Malnutrition & 32 & 9.9 \\
\hline Pneumonia and Malnutrition & 13 & 4.0 \\
\hline URTIs & 13 & 4.0 \\
\hline Malaria and Meningitis & 9 & 2.8 \\
\hline Bronchial asthma & 9 & 2.8 \\
\hline Sepsis and malnutrition & 9 & 2.8 \\
\hline Cellulitis & 8 & 2.5 \\
\hline Anemia & 8 & 2.5 \\
\hline Others & 6 & 2.2 \\
\hline
\end{tabular}

Table 2: Frequency distribution of top-10 reasons of pediatric hospital admissions at Shenan Gibe Hospital, May 2015 (n=323) 
Concerning the treatment regimens used 58 (46.4\%) of pneumonia patients were treated by combination of Gentamicin and Ceftriaxone for 10-days, followed by $32(25.6 \%)$ treated by combination of crystalline penicillin and gentamicin for 7-days. Among patients with gastro-enteritis 21(51.2\%) were treated by ceftriaxone and metronidazole for 10-days and majority of sepsis patients 35 (71.4\%) were treated by ceftriaxone and gentamicin for 7 -days while 6 (66.7\%) of meningitis patients were treated by combination of ceftriaxone + gentamicin; and $13(65 \%)$ of Cellulitis patients were treated with Cloxacillin + Gentamicin (Table 3 ).

\begin{tabular}{|c|c|c|c|c|}
\hline \multirow[t]{2}{*}{ Diagnosis } & \multirow[t]{2}{*}{ Antibiotic Treatment given } & \multirow[t]{2}{*}{ Duration } & \multicolumn{2}{|c|}{$\begin{array}{l}\text { Number of patients } \\
\text { treated with each } \\
\text { regimen }\end{array}$} \\
\hline & & & Frequency & Percent \\
\hline \multirow{4}{*}{ Pneumonia } & Gentamicin + Ceftriaxone & 10-days & 58 & 46.4 \\
\hline & Crystalline penicillin +Gentamicin & 7-days & 32 & 25.6 \\
\hline & Ampicillin + Gentamicin & 10-days & 26 & 20.8 \\
\hline & CAF + Ceftriaxone & 7-days & 9 & 7.2 \\
\hline Malnutrition & Ampicillin + Gentamicin & 15-days & 32 & 100 \\
\hline \multirow{3}{*}{ Acute gastro enteritis } & Ceftriaxone + Metronidazole & 10-days & 21 & 51.2 \\
\hline & Ceftriaxone + Gentamicin & 7-days & 14 & 34.1 \\
\hline & Ceftriaxone + Ampicillin + Gentamicin & 7-days & 6 & 14.6 \\
\hline \multirow{3}{*}{ Sepsis } & Ceftriaxone + Gentamicin & 7-days & 35 & 71.4 \\
\hline & Ceftriaxone + Ampicillin + Gentamicin & 7-days & 10 & 20.4 \\
\hline & Ceftriaxone + Metronidazole & 14-days & 4 & 8.2 \\
\hline \multirow{2}{*}{ Malaria and Meningitis } & Ceftriaxone + Gentamicin & 14-days & 6 & 66.7 \\
\hline & Ceftriaxone + CAF + Gentamicin & 10-days & 3 & 33.3 \\
\hline Pneumonia and Malnutrition & Ampicillin + Gentamicin & 14-days & 13 & 100 \\
\hline \multirow{2}{*}{ Sepsis and Malnutrition } & Ceftriaxone + Gentamicin & 14-days & 4 & 44.4 \\
\hline & Ceftriaxone + Ampicillin + Gentamicin & 10-days & 5 & 55.6 \\
\hline \multirow{3}{*}{ Cellulitis } & Cloxacillin + Gentamicin & 7-days & 13 & 65 \\
\hline & Cloxacillin & 14-days & 5 & 25 \\
\hline & Ceftriaxone & 10-days & 2 & 10 \\
\hline \multirow{3}{*}{ URTIs } & Crystalline penicillin & 10-days & 3 & 23.1 \\
\hline & Clarithromycin & 7-days & 6 & 46.2 \\
\hline & Ampicillin & 7-days & 4 & 30.8 \\
\hline Others & Sulfamethoxazole + trimethoprim & 5-days & 7 & 100 \\
\hline
\end{tabular}

Note: The above table only describes the antibiotic regimens used for treatment it is not comprehensive management of the respective illness

Table 3: Distribution of reasons of pediatric hospital admissions and their respective antibiotic treatment given at Shenan Gibe Hospital, May 2015 ( $=318$ )

Seven hundred sixty five antibiotics were prescribed for 323 patients; this indicated that 2.37 antibiotic injections per prescription were used for management of hospitalized pediatrics. Most of patients 295 (91.3\%) were treated by combination of two antibiotics and $27(8.4 \%)$ were treated with single antibiotic and 21 (6.5\%) were treated with three antibiotics. Among this antibiotic combination Ceftriaxone + Gentamicin 97 (32.8\%), followed by Ampicillin + Gentamicin 95 (32.1\%), and Crystalline penicillin + Gentamicin 38 (12.8\%) (Table 4).

\begin{tabular}{|c|c|c|}
\hline Combination of antibiotics & Frequency & Percent \\
\hline Gentamicin + Ceftriaxone & 97 & 32.8 \\
\hline Ampicillin + Gentamicin & 95 & 32.1 \\
\hline Crystalline Penicillin + Gentamicin & 38 & 12.8 \\
\hline Ceftriaxone + Metronidazole & 25 & 8.4 \\
\hline $\begin{array}{c}\text { Ceftriaxone + Ampicillin + Gen- } \\
\text { tamicin }\end{array}$ & 15 & 5.1 \\
\hline Cloxacillin + Gentamicin & 11 & 3.7 \\
\hline CAF + Ceftriaxone & 9 & 3.0 \\
\hline Ceftriaxone + CAF + Gentamicin & 6 & 2.0 \\
\hline
\end{tabular}

Table 4: Frequency Distribution combination of antibiotics used in pediatric Ward at Shenan Gibe Hospital, May 2015 ( $\mathrm{n}=296$ )

Almost all 316(97.6\%) of patients were treated with injections except for sulfamethoxazole and clarithromycin which were 
prescribed as discharge medication for 7 and 6 patients respectively. With regard to monotherapy used among hospitalized pediatric patients; cotrimoxazole/sulfamethoxazole +trimethoprim accounted for 7(25.9\%) followed by clarithromycin $6(22.2 \%)$ and cloxacillin 5(18.5\%) respectively (Figure 2).

\section{Dose of antibiotics prescribed, frequency and duration of administration}

Antibiotics were prescribed with correct dose for 294 (91\%) of followed by correct frequency and duration of administration for $280(86.7 \%)$ and 297 (92\%) of pediatrics patient respectively. By combination of dose, frequency and duration, 280 (86.7\%) of drugs were prescribed appropriately whereas 33 (10.1\%) were prescribed inappropriately (Table 5).

\begin{tabular}{|c|c|c|c|c|}
\hline & \multicolumn{2}{|c|}{ Correct } & \multicolumn{2}{c|}{ IncorrectV } \\
\hline & Frequency & Percent & Frequency & Percent \\
\hline Monotherapy & & & & \\
\hline Dose & 294 & 91 & 29 & 9 \\
\hline Frequency of administration & 280 & 86.7 & 43 & 13.3 \\
\hline Duration of treatment & 297 & 92 & 26 & 8 \\
\hline Combination of therapy & & & & \\
\hline Dose & 280 & 86.7 & 43 & 13.3 \\
\hline Frequency of administration & 280 & 86.7 & 43 & 13.3 \\
\hline Duration of treatment & 280 & 86.7 & 43 & 13.3 \\
\hline
\end{tabular}

Table 5: Dose of antibiotics prescribed, frequency and duration of administration in pediatric Ward at Shenan Gibe Hospital, May 2015 ( $\mathrm{n}=27$ )

\section{Discussion}

In this study majority of participants 197 (61\%) belongs to age group 0-4 years with mean age of pediatrics was 3.5 years. However this lower than study conducted on pediatric drug prescribing in a regional hospital in trinidad which showed that the mean age of the children was 5.52 years, with $85.6 \%$ between the age of 1 month and 5 years, and $14.4 \%$ over the age 5 years [19]. This could be explained by that the former study was done among all pediatric patients while our study subjects were hospitalized pediatrics and could also be due to difference in sample size.

Majority 245 (75.9\%) of patients had received empiric treatment while 70(24.1\%) had received for definitive therapy. This is higher than Retrospective drug utilization evaluation in pediatric patients in USA showed that large percentages (30\%-50\%) of antibiotics administered in the USA are given to prevent infection rather than to treat established disease. This practice accounts for some of the most fragment misuses of these drugs [20]. This could be due to difference in advancement in health service delivery system like advanced laboratories for diagnosis of definitive pathogens to target and expertise on antibiotics like infectious disease specialists which are at very young stage in study area.

In our study Pneumonia was single most common reason for hospitalization which accounts 125 (38.7\%) followed by Sepsis 49 $(15.2 \%)$ and acute gastro enteritis $41(12.7 \%)$. This is in line with study conducted in Jimma university specialized hospital that the major medical conditions for which antibiotics were prescribed include severe pneumonia (31.23\%) and late onset neonatal sepsis with meningitis $(20.60 \%)$ [23].

Seven hundred sixty five antibiotics were prescribed for 323 patients; this indicated that 2.37 antibiotic per prescription. Most of patients $295(91.3 \%)$ were treated by combination of two antibiotics and $27(8.4 \%)$ were treated with single antibiotic and 21 $(6.5 \%)$ were treated with three antibiotics. This is higher than study conducted in Jimma University specialized hospital showed that the average number of antibiotics per patient was 2.17 . Two hundred ten $(77.20 \%)$ of the patients were exposed to at least two antibiotics [23] and study conducted in, Hawassa showed that the mean number of antibiotics prescribed per-prescription was $1.18 \pm 0.813$ [25]. These differences may be due to variations in prescribing habits among different hospitals or among the different physicians who may explain these finding which we were unable to determine in this study and also selection of antibiotics in the previous studies were may be based on culture sensitivity testing unlike in our case which was mostly empiric and it could be due to better technological advancement and expertise knowledge in both University specialized hospitals than the study area.

In our study the most commonly used antibiotic combinations were Ceftriaxone + Gentamicin 97(32.8\%), followed by Ampicillin + Gentamicin 95 (32.1\%), and Crystalline penicillin + Gentamicin 38 (12.8\%). This similar to the case of Jimma University specialized hospital showed that the most commonly prescribed multiple antibiotic was Ampicillin plus Gentamicin (21.9\%) followed by Ceftriaxone \& Gentamicin (18.1\%) [23] And study conducted in Hawassa University referral hospital showed that 416 $(35.6 \%)$ of the prescriptions were multiple antibiotic prescriptions and from these the combination of Ampicillin and Gentamicin took the largest portion with 113(27\%), followed by Chloramphenicol and Cloxacillin 60(14.4\%) [25].

Almost all 316(97.6\%) of patients were treated with injections except for sulfamethoxazole and clarithromycin which were prescribed as discharge medication for 7 and 6 patients respectively. However this is higher than studies conducted in Dessie 
Referral Hospital showed that $76 \%$ of antibiotics were given through parenteral route with practice of parenteral to oral shift during discharge 23 (7.9\%) of cases [26]. Retrospective assessment conducted in Mekelle general hospital showed that the percentage of encounters with antibiotic injections at inpatient departments 172 (95.2\%) out of 181 sampled antibiotic prescribed were parenteral formulations [27]. This variation could be due difference in sample size, experience of prescribers and dispensers and availability of guidelines to monitor antimicrobial use.

\section{Strength and Weakness of the Study}

\section{Strength of the Study}

- The data was collected using a structured self-administered pre-tested questionnaire

- Adequate sample size was applied according to single population proportion formula

- Data collators were trained health professional

- Data quality is controlled throughout the study

\section{Weakness of the Study}

During this study there may be desirability bias

\section{Conclusion and Recommendation}

\section{Conclusion}

Referral Hospital showed that $76 \%$ of antibiotics were given through parenteral route with practice of parenteral to oral shift during discharge $23(7.9 \%)$ of cases [26]. Retrospective assessment conducted in Mekelle general hospital showed that the percentage of encounters with antibiotic injections at inpatient departments 172 (95.2\%) out of 181 sampled antibiotic prescribed were parenteral formulations [27]. This variation could be due difference in sample size, experience of prescribers and dispensers and availability of guidelines to monitor antimicrobial use.

\section{Recommendation}

Based on the finding of the study the following recommendations were made to responsible bodies

1. There is inconsistency of treatment for a given disease condition this calls government, regional health bureau to avail guidelines to monitor antimicrobial use, provision of trainings to raise the level of awareness about rational use of drugs, prescribing and dispensing of drugs through a continuous in services training, seminars and workshops to reduce use of combined systemic broad spectrum antibiotics which could lead to selection of naturally resistant strains to emerge unless intervened now "No action today: No cure tomorrow."

2. Most of patients received treatment for empiric diagnosis this tells the government and other stakeholders to play role on improving the laboratory facilities for better detection of definitive pathogen that will help to target specific pathogen during treatment

3. Practice of Parenteral administration is higher than other similar studies and there was almost negligible Parenteral to oral shifting practice, this calls for increasing professionals awareness about antibiotics particularly the benefit of Parenteral to oral conversion

4. This was cross-sectional descriptive study which will not tell factors and causality inappropriate use of antibiotics we recommend further strong studies that can give more clear insight

\section{Contribution of researchers}

Mende Mensa is senior researchers who analyzed and interpreted the findings of this study and he also prepared this document for publication. Takele Achalu conceived the study and prepared the proposal and collected data and participated in data analysis and presented the work for responsible bodies.

\section{Acknowlegments}

First of all, I would like to thank almighty God for giving us insight in my life and care and health to conduct this study.

I would like to express our deepest gratitude and appreciation to Mr. Shibiru from Jimma University Pharmacy School for his unreserved encouragement and provision of constructive comments and guidance while conducting this research.

Finally we would like to thank all staffs of Shenan Gibe Hospital, Jimma Zonal Health department and Shenan Gibe Hospital Administration for their support during data Collection.

\section{Conflicts of interest}

We have no conflict of interest during conducting this study or developing the manuscript. All expenses regarding this paper were from out of pocket of researchers. 


\section{References}

1. Tadesse S, Belay Y, Tsegaye M (2015) Antibiotics Utilization Pattern in Paediatric Ward: The Case from Tertiary Teaching Hospital, South West Ethiopia. Int J Adv Multidis Res 2: 54-61.

2. World Health Day (2011) Policy briefs. Geneva, World Health Organization, 2031. Retrieved on 9th January 2012.

3. Patrick R. Kens S, Michael A et al. (2005) Medical Microbiology agent, USA 10: 2003-212.

4. FDRE, MOH (1996) Manual of drug supply management. Addis Ababa, Ethiopia 8: 61-9.

5. Mohammed E, Muhe L, Geyid A, Dejene A, Mekonnen Y, et al. (2000) Prevalence of bacteria pathogen in children with acute respiratory infection Ethiop Med J 8: 165-73.

6. Goodman and Gilman’s (1996) The pharmacological basis of therapeutics 9th edition, USA Mc Graw-Hill.

7. Katzung B (2006) Basic and Clinical pharmacology. 7th edition. USA: Mc Graw Hill.

8. Dannis L. Eugene B. Anthony S. et al. (2006) Harrison principles of internal medicine 17th edition. USA.

9. Al-Niemat SI, Bloukh DT, Al-Harasis MD, Al-Fanek AF, Salah RK (2008) Drug use evaluation of antibiotics prescribed in Jordanian outpatient and emergency clinic using who prescriber indicators. Saudi Med J 29: 743-8.

10. von Gunten V, Troillet N, Beney J, Boubaker K, Lüthi JC, et al. (2005) Impact of an interdisciplinary strategy on antibiotic use: prospective controlled study in three hospitals. J Antimicrob Chemother 55: 362-66.

11. WHO (2002) Promoting rational use of medicines: core components, 2002 (3): 1-6.

12. Shivalella, K Jagadesh, Shreenivas, Revankar, Vedavathi H (2014) A study on prescription patterns of antibiotics in paediatric in-patients of Mc-Gann Teaching Hospital, shivamogha institute of medical sciences (SIMS) in Karnataka. IOSR J Dent Med Sci 13: P 67-71.

13. You ML, Chun Y, Yung TK, Man YH, Hsiang YC (2010) Outcomes of pharmacy interventions on pediatric medication prescribing patterns in Taiwan. Int J Clin Exp Med 2: 173-80.

14. Academy of managed care pharmacy (2009) Drug use evaluation. Asian J Med Sci 1: 88-90.

15. Bjorn B (2007) Institute of medicine center for international health university of Bergen. Antimicrobial resistance in bacterial infections in urban and rural Tanzania.

16. Antimcirobials Use, Resistance and Containment Baseline Survey Syntheses of Findings. DACA, MSH/SPS, August 2009, Addis Ababa.

17. Salih Mollahaliloglu, Alia Alkan, Basak Donertas, Senay Ozgulcu, Ahmet Akici (2013) Assessment of antibiotic prescribing at different hospitals and primary health care facilities. Saudi Pharm J 21: 281- 91.

18. Lusini G, Lapi F, Sara B, Vannacci A, Mugelli A, et al. (2009) Antibiotic prescribing in pediatric populations: A comparison between Viareggio, Italy and Funen, Denmark. Eur J Public Health 19: 434-8.

19. Orrett FA. Ghangoor E, Maharaj N (2010) Pediatric drug prescribing in a regional hospital in Trinidad. J Chinese Clini Med 5: 157-63.

20. Hing WC, Bek SJ, Lin RTP, Li SC (2004) A retrospective drug utilization evaluation of vancomycin usage in pediatric patient's. J Clini Pharm Therapeutics 29: 359-65.

21. Shivaleela, Jagadeesh K, Shreenivas Revankar, Vedavathi H, Nagaraja Prasad S, et al. (2014) A Study of Prescription Pattern of Antibiotics in Pediatric InPatients of Mc-Gann Teaching Hospital Shivamogga Institute of Medical Sciences (SIMS), Shivamogga, Karnataka 13: 67-71.

22. Amin H., et al. (2004) Antibiotic profile in Pediatric wards, Department of Child Health, Cipto Mangunkusumo Hospital. Paediatrica Indonesiana 44: 3-4.

23. Tadesse Sheleme A, Belay Y, Tsegaye Melaku K (2015) Antibiotics Utilization Pattern in Pediatric Ward: The Case from Tertiary Teaching Hospital, South West Ethiopia. Int J Adv Multidisciplinary Res 2: 54-61.

24. Mestawot Feleke, WubanteYenet, Jimma LikisaLenjisa (2013) Prescribing pattern of antibiotics in pediatric wards of Bishoftu Hospital, East Ethiopia. Inter J Basic Clini Pharm 2: 718-22.

25. Minyahil Wolde, Sultan Suleman, NestanetWorkneh, Hafta Berhan (2012) Retrospective study of the pattern of antibiotics use in Hawassa University Referral Hospital Pediatric Ward, southern Ethiopia. J Appl Pharma Sci 3: 93-8.

26. Gizework A, Seyfe Asrade A (2014) Assessment of the pattern of antibiotic use in pediatric ward of Dessie Referral Hospital, North East Ethiopia 7: 1-7.

27. Girum S, Teklemariam G (2014) Retrospective assessment of irrational use of antibiotics to children attending in Mekelle general hospital. Sci J Clini Med 3: 46-51. 